\title{
O.S.P.
}

L'orientation scolaire et professionnelle

$39 / 3$ | 2010

Travail et genre dans les médias

\section{La presse féminine : une mythologie efficace}

Succès du conservatisme ou nouveau féminisme?

Feminine Press: An efficient mythology

\section{Claude Chabrol et Mihaela Oprescu}

\section{CpenEdition}

Journals

Édition électronique

URL : http://journals.openedition.org/osp/2866

DOI : 10.4000/osp.2866

ISSN : 2104-3795

Éditeur

Institut national d'étude du travail et d'orientation professionnelle (INETOP)

Édition imprimée

Date de publication : 15 septembre 2010

ISSN : 0249-6739

Référence électronique

Claude Chabrol et Mihaela Oprescu, «La presse féminine : une mythologie efficace », L'orientation scolaire et professionnelle [En ligne], 39/3 | 2010, mis en ligne le 15 septembre 2013, consulté le 20 avril 2019. URL : http://journals.openedition.org/osp/2866 ; DOI : 10.4000/osp.2866

Ce document a été généré automatiquement le 20 avril 2019

(c) Tous droits réservés 


\title{
La presse féminine : une mythologie efficace
}

\author{
Succès du conservatisme ou nouveau féminisme? \\ Feminine Press: An efficient mythology
}

Claude Chabrol et Mihaela Oprescu

\section{Introduction 1}

1 Les débats sur la presse féminine sont bien connus, mais au fond ce qui a intéressé est surtout sa fonction sociale idéologique: relativement positive selon quelques-uns, mais plus ou moins négative pour beaucoup. De cette première question l'on passera trop vite à la seconde: cette presse très présente pourrait sans doute agir aussi sur les comportements des femmes en influençant leur esprit. En somme, elle aurait une fonction propagandiste en faveur d'une division encore traditionnelle des tâches, des rôles et statuts selon un marquage encore traditionnel du genre. Dans cet article, on se donnera d'abord pour but de clarifier les positions en montrant les postures distinctes des mouvements féministes engagés et des chercheurs spécialisés, entre autres ceux des cultural studies. On entend ainsi rappeler la complexité de la discussion et les partis pris comme les options théoriques et méthodologiques engagés. Il n'est pas évident de réduire la variété des articles et des magazines de cette presse aux exemples qu'on en extrait en sous-estimant ceux qui vont dans le sens contraire ou en minorant leur rôle, mais cet enjeu ne pourrait devenir pertinent que sous certaines conditions plus théoriques que statistiques, en réalité.

2 Selon nous, il importe en effet de produire d'abord un modèle et un type pour orienter l'analyse descriptive empirique. On connaît les distinctions liées aux notions d'idéologie et de mythologie. Cette presse fonctionne-t-elle surtout comme un outil idéologique à peine masqué face à la contre-idéologie des mouvements sociaux inspirés par les actions des féministes ou est-elle d'abord et avant tout un espace mythologique, au sens de Lévi-Strauss, 
de conjonction des oppositions sociales et mentales ordinaires liées au genre, ce qui n'exclurait pas des fonctions et usages secondaires? En tout cas, les effets d'une idéologie et d'une mythologie ne sont pas du même ordre sur la textualité.

On présentera dans un premier temps un panorama succinct des recherches inspirées par les principaux courants féministes, en particulier en France, pour les confronter ensuite à leurs développements et leurs problématisations dans les cultural studies anglo-saxonnes. Ce faisant, ce qui sera progressivement questionné est d'abord l'hypothèse, souvent posée mais peu argumentée, qui ferait de cette presse un outil puissant d'action directe ou indirecte sur des esprits très passifs. Celle-ci renvoie à une option psychosociologique théorique fort discutée en psychologie comme par les courants des cultural studies qui soulignent au contraire l'importance de l'activité engagée en réception. On verra en outre que la confusion regrettable entre catégorisations sociales, en général implicites et peu conscientes, et stéréotypisations explicites, rend plus difficiles les discussions.

Pour une approche alternative à cette problématique très conceptuelle, nous présenterons dans un second temps une recherche empirique 3 qui montre au sein d'un même milieu d'étudiantes du même âge la force de leurs orientations psychologiques opposées (BSRI de Bem) dans la réception d'articles de presse féminine. Ces derniers sont choisis par des juges de la même population pour leurs orientations contraires («féminisante» vs «non féminisante»). La clarté des résultats devrait permettre d'engager de nouveaux débats et en tout cas renforcer la thèse de ceux qui plaident pour une conception beaucoup plus complexe du travail psychique activé en réception.

\section{Les analyses féministes et les orientations contre- idéologiques}

5 Pour certains analystes, cette presse répondrait d'abord à une logique de succès commercial; mais celle-ci demanderait elle-même à être expliquée. La grande majorité des lectrices de cette presse seraient des femmes qui aimeraient se retrouver, certes sans y être contraintes, dans un univers voué enfin totalement à la femme, en feuilletant les pages d'un produit médiatique, considéré malgré son succès et sa diversité réelle comme «mineur» et rarement étudié de façon systématique en France par les sciences sociales et du langage à l'opposé des journaux d'information ou des productions télévisuelles.

6 «Le» discours de la presse féminine (mais n'y en a-t-il qu'un seul, et faut-il négliger les différences d'âge et de classes sociales qui en constituent la diversité?) comme «le» discours médiatique en général, traduirait en les reformatant les représentations collectives dominantes socialement ( $\mathrm{du}$ public «manipulé», des journalistes, des publicitaires?) et non peut-être celles qui sous-tendent les rapports sociaux effectifs des hommes et des femmes.

\section{La critique féministe libérale: décalage des stéréotypes et des normes idéales de genre}

7 Ce courant se préoccupe notamment de l'existence des stéréotypes et de leur influence sur la formation de l'identité de genre et sur le processus de socialisation de genre ${ }^{2}$, dénonce le décalage entre représentations véhiculées et comportements, et en fait porter une bonne part de responsabilité à l'action «propagandiste» des médias, comme si cette 
presse pouvait les faire coïncider pour la première fois dans l'histoire des cultures. Cette critique propose en effet un type d'analyse qui pose ou suggère une quasi relation de causalité et non une simple corrélation entre les messages de genre et les effets que ces messages produiraient, idéalement ou réellement (?), sur les mentalités, les attitudes et les comportements des récepteurs.

Les messages contenant des stéréotypes traditionnels de genre auraient pour ces courants des conséquences sur la formation identitaire et sur la production des rôles de genre. En outre, les lectrices seraient supposées recevoir toutes plus ou moins de la même manière les messages de genre, quel que soit le contexte de la réception, leurs facteurs motivationnels et psychologiques ou leurs caractéristiques sociales et culturelles. On perçoit ici sans doute la continuité d'une tradition de dénonciation «contre-idéologique» qui s'inscrit dans une histoire du concept d'idéologie que le marxisme a inauguré dès le XIX ${ }^{e}$ siècle et que des mouvements critiques divers ont développé à leur façon, en accentuant beaucoup plus le pouvoir autonome des superstructures aujourd'hui. Toutes ces critiques contre-idéologiques doivent recourir explicitement ou non à des notions aussi délicates à préciser et manier que l'«aliénation» ou la «fausse conscience», pour expliquer un aveuglement qui frapperait très différemment les membres de groupes restreints ayant une histoire et une vie commune et composés de membres d'origine sociale et économique assez similaire, sauf exceptions.

9 Ces idées, en tout cas, sont bien rassemblées dans le postulat «féministe», pour lequel toute femme serait victime de l'influence des médias dans les processus d'identification, de comparaison sociale ou dans l'acquisition et le maintien de l'estime de soi. Avec la publication, en 1963, de La femme mystifiée de Betty Friedan, un mouvement critique à l'égard des médias se développe aux États-Unis. The feminine mystique a marqué la naissance d'un mouvement visant à réévaluer le rôle des femmes dans la société américaine. Celui-ci n'est pas seulement engagé dans une lutte matérielle pour l'égalité des droits pour les femmes, mais également dans un conflit symbolique concernant les définitions, entre autres véhiculées par les médias, de la «féminité» et de la «masculinité». Ces définitions seraient héritées des codes symboliques dominants. Les médias y sont présentés, souvent de manière intuitive ${ }^{3}$, comme l'un des vecteurs les plus influents de la transmission de ces codes, sans doute parce que le plus visible, mais aussi le moins chargé de prestige, contrairement à la littérature ou à la peinture, sans parler des arts populaires trop bien investis (cinéma, chansons à succès) ou de l'école ou des relations familiales, trop proches peut-être de l'expérience de chacun. Les pratiques médiatiques de genre sont de ce fait devenues la cible préférée des mouvements des femmes. On enjoint aux médias de présenter une image plus positive de la femme, dans des rôles plus modernes. La critique féministe des médias relève l'usage redondant des stéréotypes de genre et l'utilisation d'images «réductrices» ou «déformées» de la femme dans ces médias.

10 Les fondements de la critique féministe des médias sont à rechercher dans les mouvements contre-idéologiques (voir Louis, 1997) qui ont tenté de réhabiliter la place de la femme dans la société, et de changer ses représentations dans les supports culturels. Le féminisme situe la cause de l'oppression des femmes au niveau de la structuration sociale. Mais celle-ci a été identifiée de manière différente par plusieurs courants, soit sous la forme d'une société capitaliste, sexiste ou patriarcale ${ }^{4}$.

11 En fait, le féminisme se manifeste dans des courants divers: «socialiste», «libéral» ou «radical». D'après une classification de la critique féministe proposée par Steeves (1987) 5: 
12 - la critique « radicale » s'intéresserait prioritairement à la pornographie et à l'influence des images violentes sur les comportements des hommes envers les femmes ;

13 - la critique « libérale » se préoccuperait surtout de l'existence des stéréotypes et de leur influence sur la formation de l'identité de genre et sur le processus de socialisation de genre ;

14 - tandis que la critique "sociale ou marxiste " mettrait l'accent sur l'interaction entre le genre, la classe, et l'idéologie et sur la structure capitaliste véhiculée par les médias. Epstein (1978), théoricien féministe marxiste parle de la relation entre la marginalité et la dévalorisation des problématiques féminines et le rôle joué par la presse dans la (sous)médiatisation de ces thématiques (voir Van Zoonen, 1991).

15 Les aspects abordés dans les analyses de contenu entreprises par la critique féministe classique des médias (voir Steeves, 1987), sont les représentations «inégales» des rôles de genre, l'invisibilité des femmes dans les médias, et l'exploitation de l'image de la femme. Les médias y sont toujours considérés, et ce de manière toujours intuitive, comme un des vecteurs influents de la transmission des rôles de genre, à travers des images stéréotypées mais sans mention des éléments contre-stéréotypés pourtant présents. Ces stéréotypes seraient responsables non seulement du maintien d'une image dévalorisante et incomplète de la femme, mais aussi d'une influence néfaste sur les comportements associés aux rôles de genre (voir Epstein, 1978). Leurs effets sont supposés néfastes, immédiats et extrêmement puissants, agissant à travers un processus d'imitation, assez daté conceptuellement, que mettraient en œuvre les récepteurs, notamment les femmes confrontées à ces «clichés discursifs» dévalorisants et non conformes à leur réalité.

16 Ces analyses de contenu aboutissent en général à une critique totale: lorsqu'un modèle de genre est repérable dans les médias, on l'interprétera comme un indice du schéma dominant de soumission des femmes, car hommes et femmes imiteraient quasiautomatiquement les modèles de genre présentés par les médias. Dès ses débuts, cette discipline, qui s'était dénommée "études de l'image de la femme», sélectionnait dans la presse surtout des icônes de femmes considérées comme trop limitatives, car souvent cantonnées au seul aspect esthétique. Des images de femmes jeunes, belles, dénudées et adoptant des postures lascives, quasi invariables, constitueraient la norme de la représentation dominante, en particulier dans la publicité. Les femmes, même dans les territoires peu conformes à leurs domaines de compétences traditionnels, resteraient encore trop séduisantes.

17 Ces images, parfois appropriées dans le domaine du soin et de la beauté ou celui de la mode, seraient «inacceptables» dans d'autres types de lieux médiatiques. Que ce soit dans l'univers de la mode ou de la publicité où un seul type de femme est supposé acceptable (mince, voire anorexique et grande), ou au cinéma, où le succès d'un long métrage ne dépendrait souvent que de la seule beauté physique de ses jeunes comédiennes, la question de la normativité réductrice des attributs physiques est soulignée. L'argument féministe reposerait sur le fait que l'image médiatique ne correspond pas ou plus à la situation réelle actuelle, dans laquelle un nombre important de femmes travaillent, occupent des postes à responsabilité et ne se préoccupent pas exclusivement de leur maison, de leur famille ou de leur apparence. Il y aurait un retard culturel (cultural lag) des croyances et des attitudes envers les femmes par rapport à la réalité. La solution proposée, produire et transmettre par un contre-discours des images plus réalistes des femmes, même si elle était légitime, resterait néanmoins peu évidente. Les aspects 
abordés dans les analyses de contenu entreprises par la critique féministe classique des médias (voir Mouffe, 1992), sont les représentations «inégales» des rôles de genre, l'invisibilité des femmes dans les médias, et l'exploitation de l'image de la femme. Les médias y sont toujours considérés, et ce de manière toujours intuitive, comme un des vecteurs influents de la transmission des rôles de genre, à travers des images stéréotypées mais sans mention des éléments contre-stéréotypés pourtant présents.

La critique féministe des médias perçoit le genre comme une variable plus ou moins stable, facilement identifiable et qui, par conséquent, devrait être représentée correctement et équitablement. Une telle conceptualisation de la variable sociale de genre est profondément problématique, car elle ignore ou dénie la nature dynamique du genre, ses spécificités historiques et culturelles, ainsi que ses contenus et ses interprétations contradictoires. Il n'y a pas véritablement de critères fixes évidents selon lesquels les médias devraient représenter les acteurs sociaux en termes de genre. L'identité de genre est considérée par la critique féministe comme socialement construite ce qui impliquerait qu'elle est d'abord un produit des circonstances et des opportunités sociales et psychologiques variables. Or la critique féministe française des médias demeure aujourd'hui majoritairement dénonciatrice, et reste souvent cantonnée au niveau de la source productrice de messages dits «réducteurs», "stéréotypés» ou «sexistes». Sans remettre en question les motivations et les fonctions de ce type de critique axée prioritairement sur la source et le message, on pourra penser qu'elle s'apparente davantage à une campagne politique féministe, dont on ne peut nier l'utilité empirique pour des combats contre-idéologiques mais dont il faut bien souligner la limitation pour l'analyse psycho-sociale et langagière. Ainsi pour Debras (2003), spécialiste en sciences du langage et rédactrice à La Terre de chez nous:

Les thèmes traités dans la presse française répondent à un standard masculin, pire,

à un standard masculin dépassé. Il n'y a pas de place pour les femmes, ni dans la presse dite féminine qui les renvoie à l'image de la potiche, ou de la superwoman, ou de la femme nourricière à la Rousseau, ni dans la presse d'information générale où elles existent peu, et parfois bien mal. Et quand je leur pose la question: Mais pourquoi est-ce que vous continuez à acheter cette presse féminine pour laquelle vous n'êtes pourtant pas tendres? Elles me répondent: Parce que là, au moins, on parle de nous. (p.179) (voir Butler, 2005)

Bref, la manière dont ces médias traitent des femmes serait, d'après les chercheurs de la critique féministe des médias, stéréotypée et réductrice. Néanmoins, une telle argumentation apporte encore très peu d'informations sur la manière dont les représentations «sexistes» sont perçues et interprétées par les femmes elles-mêmes, ainsi que sur le véritable impact sur les mentalités et les comportements sociaux; or de nombreux travaux montrent bien les rapports complexes que les lectrices ont avec leur presse:

Les chercheures jugent aliénante la consommation médiatique privilégiée par quantité de femmes. Plusieurs affichent un malaise évident devant leurs observations, surtout devant le plaisir véritable que ressentent les femmes à consommer des produits jugés insignifiants [...]. (Plante, 2004, p.120) (voir Brown, 1990)

20 La question de la sous-représentativité en termes de domaines de compétence est un autre aspect mis en avant par la critique féministe. Les femmes seraient moins visibles que les hommes, moins bien mises en valeur, moins crédibles. Quel serait alors le rôle de la femme? Goffman (1977?) ${ }^{6}$ avait suggéré que la femme était utilisée le plus souvent comme objet attractif et distractif. Il est en effet exact qu'une source attractive a une 
influence positive sur la réception du message (voir Busby, 1975) et pourrait même influencer le mode de traitement d'un message. L'attractivité de la personne en ellemême est un indice périphérique qui pourrait faciliter, en suscitant l'attention, un traitement en profondeur, plus systématique (voir Dominick, 1979). La femme séduisante, supposée trop facilement docile, dont l'apparition n'a aucun rapport avec le produit vanté, est le plus souvent utilisée afin d'attirer l'attention, en tant qu'élément de distraction positive. Tel serait souvent l'apanage de la publicité, qui, pour mieux vendre et influencer son public, utiliserait la femme comme simple source d'attrait.

21 À partir de l'observation des corpus d'annonces, plusieurs chercheurs (Guyon, 1984; Herne, 1993) ont montré que les personnages des publicités sont très majoritairement associés à des rôles spécifiques à leur sexe: aux hommes la sphère publique, aux femmes la sphère privée. Il y aurait une certaine infériorité des femmes qui s'exprime à travers une multiplicité des signes. Les hommes sont sur-représentés dans les emplois qualifiés, correspondant à un haut niveau de diplômes, et dans des rôles actifs et dominants. Les femmes, en position assistée, subalterne, n'apparaissent que rarement, ou à titre décoratif, dans les mondes masculins (travail, automobile, sport, services). Elles sont, au contraire, surreprésentées dans les secteurs domestique et de la beauté.

Une limite de ces travaux, soulignée par Perret (2003), est leur tendance à tomber souvent dans une critique systématique. Lorsqu'un modèle de genre est repérable dans le traitement publicitaire, on l'interprétera comme indice du schéma dominant, visant à la soumission des femmes, mais quand une annonce inverse le rapport traditionnel de pouvoir et l'attribue à une femme, ce qui survient certes de façon encore minoritaire, on dira que «l'inversion du modèle de [domination] en est clairement sa justification scandaleuse» (Louis, 1997, p.30)?!

Quand une annonce montre des images alternatives de couples égalitaires, ce sont pour «des marques de grand luxe qui contribuent ainsi à amalgamer richesse et tendresse, laissant aux pauvres le monopole de la violence» (Louis, 1997, p.31). Les recherches militantes s'interdisent ainsi souvent de saisir les évolutions et les nuances du phénomène qu'elles dénoncent. Elles s'empêchent surtout de comprendre les raisons de cette domination autrement que par la dénonciation de facteurs supposés, tels que la «misogynie des publicitaires» dont pourtant le but ne devrait être que de séduire pour vendre; alors pour quelles raisons profondes préfèreraient-t-ils plus souvent des arguments supposés décalés et par là risqués (voir Debras, 2003)? Ce qui pouvait être compréhensible culturellement de la part du clergé catholique ou des politiques conservateurs jusqu'aux années 60 au $\mathrm{XX}^{\mathrm{e}}$ siècle, ne demeure-t-il pas bien obscur en ce cas?

24 La presse féminine représente un des produits médiatiques les plus dénoncés par la critique féministe, sans doute en raison de la très grande popularité dont elle bénéficie auprès des femmes et en raison des innombrables représentations, images et modèles féminins qui y sont associés.

Les magazines féminins représentent l'une des institutions sociales les plus importantes de notre temps [...] ces magazines contribuent à la construction de l'image de soi de la femme et de l'image sociale de la femme. (Ferguson, 1983, p.1) (voir Plante, 2004)

Selon Ferguson, la presse féminine aurait le pouvoir d'influencer les comportements de rôle de genre. Les occurrences discursives et visuelles des rôles de genre sélectionnées sont supposées ne pas traduire fidèlement la réalité sociale et sont donc dénoncées 
comme limitatives, stéréotypées. Les normes de genre constituent donc des prescriptions concernant les comportements et les caractéristiques «appropriés», ici et maintenant, pour les hommes et pour les femmes à ce moment donné de l'histoire de nos sociétés. Les forces conservatrices des stéréotypes et novatrices des contre-stéréotypes rempliraient un rôle de soutien et de justification dans la chaîne des processus par lesquels des relations entre les sexes sont maintenues. Les stéréotypes de genre, dérivés mais aussi soutiens actifs de la division sexuelle du travail, renforceraient, à travers des croyances normatives, la tendance des individus à se conformer à cette division dans une réalité, peu souhaitable, mais bien existante.

\section{Les courants des culturals studies: les nouveaux paradigmes}

26 À côté de ces trois courants dominants de la critique féministe des médias, les cultural studies anglophones et les queer studies adopteront donc des procédures assez divergentes dans l'analyse des médias: en lieu et place d'une analyse de contenu rapide et dénonciatrice pour les «féministes» (voir Goffman, 1977), des analyses de discours culturalistes et compréhensives en réception pour les cultural studies. Ces derniers mettent en œuvre un type particulier d'analyse des médias, en proposant un changement de paradigme, qui s'écarte de l'universalisme ou du différentialisme classique pour proposer une conception dynamique qui prendra en compte le rôle actif du récepteur, supposé passif par les courants précédents.

Les courants «féministes» et les cultural studies (voir Petty \& Cacioppo, 1986) adoptent donc des procédures assez divergentes dans l'analyse des médias: en lieu et place d'une analyse de contenu rapide et dénonciatrice pour les féministes (voir Chaiken \& Maheswaran, 1994), les analyses de discours des culturalistes seront plus orientées vers le travail décisif du récepteur pour les cultural studies. Dans ce modèle, ce changement de paradigme passe essentiellement par l'étude de l'activité du récepteur dans la transmission des messages de genre.

Les chercheurs culturalistes considèrent que le pouvoir de représenter des réalités symboliques à travers les médias n'appartient pas ou pas exclusivement aux systèmes hégémoniques de domination (sociale, politique, masculine), mais également aux récepteurs. Les récepteurs prennent le pouvoir de se représenter la réalité et de construire le sens de cette réalité subjective en termes de classe sociale, de genre, de race, de position sociale à l'intérieur d'un groupe donné. L'idée que les productions médiatiques puissent être jugées comme des reflets ou surtout des distorsions de la réalité va laisser la place à une approche de la réception active des médias, une approche qui se place non pas au-delà, mais "du côté» de l'audience. Le moment de la réception devient ainsi la clé pour la compréhension des effets de sens que les femmes attribuent aux messages, et le contexte de la réception, psychologique, discursif, social ou de classe, détermine la direction et la puissance de la signification.

Ce modèle théorique est conceptualisé dans l'espace anglophone (presque exclusivement) par des chercheurs féministes comme Hobson (2003), Radway (1987), Shields (2002). Pour elles, il n'est plus aussi évident de considérer le genre comme un facteur déterminant ou fondateur des relations humaines et sociales. Des facteurs comme l'ethnie, la sexualité, la classe sociale, interagiraient fortement sur la notion de genre. Dans cette perspective, la critique féministe post-structuraliste rejette l'essentialisme et le déterminisme de type marxiste ou radical. La perspective essentialiste de la catégorie «femme» est écartée et le 
débat se déplace vers la question suivante: comment se construit la catégorie «femme» dans les différents discours, dont celui de la presse féminine?

Les théoriciens des queers studies comme Chantal Mouffe militent pour un antiessentialisme et pour une déconstruction de l'entité «femme», non qu'elle soit caduque, mais parce qu'il faudrait l'appréhender comme un espace discursif, ouvert et indéterminé. Le genre ne devrait plus être considéré comme un facteur de définition des expériences humaines:

Il n'y a pas de raison que les différences sexuelles soient pertinentes dans toutes les relations sociales. Aujourd'hui, de nombreuses pratiques sociales, discursives et institutionnelles construisent les identités masculines et féminines différemment et la distinction masculin-féminin est considérée pertinente dans beaucoup de domaines. Mais cela ne signifie pas que les choses doivent rester ainsi, et on peut très bien imaginer que les différences de genre deviennent non pertinentes dans beaucoup de relations sociales. (Mouffe, 1992, p.377) ${ }^{7}$

La pensée queer suggère que le genre est une question de performance, ou de performativité, un rituel plus ou moins normé de gestes et de comportements assignés socialement aux individus des deux sexes. Selon Butler (2005) la "performativité» de genre est une assignation normative (voir Ferguson, 1983). C'est ainsi que nous sommes constitués en tant que sujets: le genre n'est pas notre essence, qui se révélerait dans nos pratiques, ce sont des pratiques situées du corps dont la répétition instituerait le genre et les représentations occurrentes.

\section{Catégorisations et stéréotypages «utiles»}

32 Face aux courants qui insistaient sur l'idée d'une mise en circulation de représentations «fausses» (torsion, inversion idéologique), des chercheuses comme Eeagly et Diekman (1997) soulignent plutôt l'exactitude et le réalisme des catégorisations de sexe proposées qui apparaissent. Celles-ci produisent en effet des descriptions généralement «véridiques», donc fiables en situation, des différences entre groupes de sexe (traits de personnalité différents et auto-descriptions). Elles attribuent ces résultats à la fois au nombre d'observations croisées entre hommes et femmes dans leurs rôles sociaux respectifs et à leur utilisation massive dans le monde du travail et les médias. On devrait y ajouter leur qualité cognitive car ces catégorisations permettent une économie remarquable de prédictibilité puisqu'elles pré-structurent le système des attentes normatives et prescriptives croisées de rôles de genre. En somme les acteurs sociaux féminins confirmeraient souvent par leurs conduites les catégorisations de genre (Snyder, 1981) et ce pour répondre aux attentes que les autres, hommes ou femmes, sont supposés avoir par rapport à leur comportement (Selffulfilling prophecy) (voir Chabrol, 2007).

Cependant, il importe maintenant de faire une distinction plus nette entre stéréotypes et catégorisations sociales. En psychologie sociale, le stéréotype désigne l'ensemble des catégories dans lesquelles nous parlons explicitement des autres. Les stéréotypes constitueraient un mécanisme de maintien explicite des préjugés. Alors que les catégorisations sont des attitudes évaluatives qui comportent un cadre d'expression très large et souvent peu explicite et peu conscient, les stéréotypes se traduisent généralement par des conduites explicites verbales publiques. Si le stéréotype se caractérise par son uniformité, la catégorisation a un caractère d'appréciation plus vaste qui intègre un ensemble de fonctions diverses, relatives aux rapports des races, des sexes, des religions ou des classes sociales. Les catégorisations prouvent le caractère structural 
et dynamique des représentations sociales mises en jeu dans les rapports sociaux, tandis que les stéréotypes montrent surtout leur caractère sémiotique fonctionnel dans les relations interlocutives et discursives.

Cette distinction est judicieuse dans l'analyse des stéréotypes de genre transmis par les médias. Les stéréotypes médiatiques des rôles de genre sont considérés dangereux et réducteurs. La solution proposée par la critique féministe est une réduction massive de ces stéréotypes. Moins de stéréotypes et plus de contre-stéréotypes conduiraient à un affaiblissement des normes de genre, à la diminution du sexisme envers les femmes, et à l'égalité entre les individus. Cependant, ne plus utiliser des stéréotypes de genre dans les messages médiatiques ne signifierait en aucun cas que le processus de catégorisation sociale, lui, cesserait de fonctionner et d'assurer, en accord avec les normes sociétales, la persistance des attitudes mises en cause, ainsi que le montre la persistance du racisme, lui, légalement exclu des discours publics dans la société française contemporaine ou du sexisme dans les sociétés communistes après guerre, étrangement absent des analyses, même historiques.

Le stéréotype ne serait que l'actualisation discursive de catégories liées à des processus psychiques (voir Chabrol, 2007) qui subissent de nombreuses transformations pour pouvoir être sémiotisées dans les discours circulants (accentuation, atténuation, disparition, retournement en son contraire, transformations liées aux genres discursifs d'arrivée...).

Pour la critique féministe, on l'a vu, la simple exposition aux stéréotypes de la presse féminine renforcerait les normes sociales de la division des sexes, au détriment de l'émancipation des femmes. En revanche, pour la critique de type culturaliste, l'exposition à ce type de message n'apporte aucune réponse concernant l'intensité des effets des médias. Les lectrices adapteraient les contenus de cette presse et surtout leur usage à leur propre environnement cognitif, affectif et social.

En conséquence, on ne s'étonnera pas que la recherche sur les récepteurs (audience research) soit devenue progressivement une discipline autonome de la critique des médias, représentée par des chercheurs comme Janice Radway (1987). La critique appelée new audience research est associée au chercheur culturaliste David Morley, et à son livre, paru en 1980, The Nationwide Audience. Ces approches s'inspirent de la théorie de la «lecture négociée» de Hall (1980) qui introduisait l'idée d'un récepteur actif qui négocierait le sens d'un message médiatique en fonction de ses attentes, de ses buts, et de ses motivations, ce que confortent les travaux de la psychologie sociale de la persuasion développés par Bandura et Ajzen (cf. Chabrol \& Radu, 2008).

Les approches communicationnelle et discursive de l'étude des médias proposent le terme de «contrat» de lectorat, contrat qui engage producteurs et récepteurs dans un processus de co-construction de sens (voir Charaudeau, 1994; Ghiglione \& Chabrol, 2000). De ce point de vue, les femmes-lectrices s'investiraient dans l'acte de la réception de la presse féminine, en ayant des attentes, des croyances, des préférences préalables par rapport à ce type de message et par rapport à son contenu argumentatif, et en y apportant leurs propres interprétations.

39 La réception de ce type de presse ne se produit pas dans un vide psycho-social, elle est régie fondamentalement par des déterminants psychologiques de personnalité, en interaction avec des facteurs socioculturels. La réception de la presse féminine est effectuée par un public féminin qui a conscience du contrat et des possibilités de 
négociation de ce contrat lors de la lecture. Les lectrices modulent leurs attentes par rapport au contenu et à la forme du message d'un article ou d'une publicité, selon le contrat de divertissement et de détente que présuppose la lecture d'un magazine de beauté/mode féminin et co-produisent les significations qui leur importent comme tout lecteur en réception (Chabrol \& Vrignaud, 2006, pp.135-156).

Ce positionnement théorique appelle des preuves empiriques, mais l'ampleur et la complexité des problèmes rendant délicat l'usage d'emblée d'une enquête extensive, on lui préférera une étude de taille réduite de type quasi expérimental.

\section{Une recherche pour mieux comprendre}

\section{Objet}

41 Nous avons réalisé une étude en réception de la presse féminine, par des jeunes femmes, en tentant de prouver que la réception de messages médiatiques varie d'abord selon leur personnalité sociale soit traditionnelle ou «féminine», soit «masculine», c'est-à-dire en congruence avec les luttes et changement sociaux récents et leurs interprétations par les mouvements dits «féministes» (Oprescu, 2008). Cette étude s'appuie sur la théorie de l'androgynie psychologique de Sandra Bem, psychologue et féministe, qui affirme qu'il y aurait des femmes «féminines», des femmes «androgynes» et des femmes «masculines», selon leur "genre psychologique». Nous avons supposé que l'évaluation d'un article de presse et de la presse féminine en général, ainsi que l'évaluation de l'influence de cette presse sur l'image de soi des femmes devraient donc varier significativement en fonction de leur genre psychologique radicalement opposé.

\section{Méthode}

Cette étude propose de confronter des femmes de genres psychologiques très différents à des articles «stéréotypés» et "contre-stéréotypés», afin de dégager des différences nettes dans leur évaluation des messages et dans leur identification avec les modèles féminins proposés. Deux groupes de sujets «féminisants» sont confrontés à des messages sur «le corps» et sur «l'âge», en accord pour l'un des deux ou en désaccord pour l'autre avec leur orientation supposée et de même pour les deux groupes de sujets «non féminisants». L'orientation des sujets a été identifiée à l'aide d'un questionnaire de personnalité, le BSRI (Bem Sex Role Inventory), proposé par Bem (1974).

Participants. 246 étudiantes en communication ont été interrogées afin de sélectionner des sujets ayant des orientations de genre différentes: «féminines» ou «masculines». 209 étudiantes ont répondu à une consigne d'auto-description, le BSRI, en version complète, avec les 60 items (20 féminins, 20 masculins et 20 neutres) validés par Bem (1974). Les participantes étaient des jeunes étudiantes en sciences humaines de Paris 3 (166 participantes) et en sciences exactes de Paris 6 (43 participantes). Nous leur avons demandé de se décrire le plus fidèlement possible, le plus rapidement possible, telles qu'elles pensaient être et non pas telles qu'elles voulaient être. À partir des résultats au questionnaire de Bem, deux groupes de sujets «extrêmes» ont été constitués: un groupe de 25 «féminines» (13 d'entre elles ayant passé la version à orientation «féminisante», et 12 la version «masculinisante») et un de 24 «masculines» (12 d'entre elles ayant passés la version à orientation «féminisante», et 12 la version «masculinisante»). 

«contrat» de lectorat.

Matériel. Entre janvier 2005 et avril 2007 nous avons sélectionné des articles de presse féminine dans plusieurs mensuels ou hebdomadaires (Elle, Marie France, Marie Claire, Biba, Cosmopolitan, Gala, Femme Actuelle, Avantages) de genres journalistiques différents: éditorial, lettre du lecteur, revue de presse (opinions ou commentaires), chronique spécialisée, compte rendu, interview, analyse, reportage, dossier, portrait, enquête (faits rapportés ou expliqués). Le choix des articles a été orienté vers les occurrences des stéréotypes de beauté corporelle et des occurrences des stéréotypes liés à l'âge (jeunesse).

La thématique «telle que l'on veut être» semble s'opposer à celle du «telle que l'on croit être». L'appréciation des femmes de leur propre corps semble osciller entre idéal et efficacité personnelle. En général, les femmes évaluent négativement leur apparence réelle par rapport à l'apparence idéale. Notre choix s'est logiquement dirigé vers les articles contenant des représentations corporelles des femmes comme thématique usuelle et familière à toutes les lectrices des magazines féminins du point de vue du

Une autre thématique apparaît souvent: celle de l'âge. Il s'agit d'affronter et de masquer les premiers signes d'âge, de changer ou de modifier les comportements en fonction de l'âge ou bien de renoncer ou de choisir des loisirs «appropriés» à son âge. Nous avons sélectionné des articles du même genre, «Dossiers» dans Biba, Elle et Marie France. Nous y avons choisi ${ }^{8}$ deux versions, stéréotypées («féminisante») et contre-stéréotypées («non féminisante»), qui s'organisaient de manière similaire, autour des témoignages de femmes sur leurs rapports au corps et sur leur vision de l'âge.

Dans la version «féminisante», stéréotypée de l'article sur le corps, le titre était «Ce corps, mon meilleur ennemi!» (Marie France, février 2006, p.87), tandis que dans la version «non féminisante», le titre de l'article était «Réconciliée avec mon corps» (Biba, 2006, 135, p.78). Les sous-titres des deux versions sont également différents du point de vue des arguments. Dans la version «féminisante», l'argumentation va dans le sens d'une preuve scientifique de l'insatisfaction corporelle de la majorité (71\%!) des femmes: «La principale crainte des Françaises pour l'avenir: grossir (71\%), quand 70\% ont peur d'être exclues de la société et 50\% d'être quittées» (Marie France, février 2006, p.87).

La version «non féminisante» de l'article sur le corps propose un sous-titre d'un tout autre type d'argumentaire: «Si on apprenait à se regarder et à s'aimer telle qu'on est (et non telle qu'on croit être), on n'entamerait pas une vraie révolution dans notre vie? Si. Et après révolution, il y a quoi? La libération!» (Biba, 2006, 135, p.78).

La même perspective argumentative oppositionnelle sous-tend les versions «féminisante» et «non féminisante» de l'article sur l'âge. Dans la version stéréotypée, l'âge est un facteur d'angoisse, de stress, qui demande une préparation psychologique et une réorganisation de la vie quotidienne: «À quoi voit-on qu'on a 30 ans? 30 ans chez les filles c'est aussi la dernière ligne droite pour devenir femme [...] on commence à faire une croix sur ses rêves de jeunesse» (Elle, novembre 2006, 27, p.133).

$\mathrm{Au}$ contraire, dans la version non féminisante de l'article sur l'âge, 30 ans c'est un moment de prise de conscience de sa propre individualité, de son indépendance et, paradoxalement, de sa jeunesse: «30 ans: j’ai mis longtemps à devenir jeune. [...] Fringues, sorties et même idées... à 30 ans on s'autorise à faire, à être, à penser tout ce qu'on a zappé à 20 [...] on s'autorise à être adulte» (Biba, 2006, 114, p.144). Ajoutons, au passage, que l'on trouve dans tous ces médias des articles orientés dans l'un ou l'autre sens et qu' 
Elle ou Marie France ne sont pas réputés pour leur orientation exclusivement «féminisante», ni Biba pour le contraire.

51 Procédure. Une procédure classique d'évaluation par des juges de la même population nous a permis de déterminer l'orientation «féminisante» ou «non féminisante» de chaque article. Après la validation de l'orientation textuelle, nous avons proposé quatre versions de livrets pour une étude en réception de l'impact des messages stéréotypés ou contrestéréotypés de la presse féminine. Chaque livret contenait deux articles, âge et corps, de la même orientation textuelle, «féminisante» ou «non féminisante». Nous avons présenté les quatre versions de livret aux participantes, jeunes femmes étudiantes, lectrices habituelles des magazines sélectionnés.

Hypothèses. Celles-ci prévoient une correspondance entre l'orientation psychologique de genre mesurée avec le BSRI et l'évaluation de la presse féminine et des messages véhiculés dans cette presse. Nous nous attendons donc à retrouver une relation claire entre le genre psychologique et la direction évaluative, positive ou négative des articles stéréotypés et contre-stéréotypés.

L'hypothèse (H1) supposait que les participantes de genre psychologique «masculin» évalueraient plus positivement les articles sur «le Corps» puis sur «l'Âge», dans leur version contre-stéréotypée, non féminisante, en s'identifiant avec les problématiques, les modèles et les représentations proposées des versions «non féminisantes» de chaque article. Par contre, l'hypothèse (H2) posait que les participantes «féminines» intérioriseraient plutôt les stéréotypes féminins et s'identifieraient davantage aux modèles et représentations des versions stéréotypées de ces articles. Les participantes «féminines» devaient donc juger plus favorablement les articles d'orientation «féminisante», tandis que les femmes «masculines» devaient juger plus favorablement les articles «non féminisants». Les femmes «féminines» devaient s'identifier davantage avec les femmes présentées dans la version «féminisante» et juger plus favorablement les modèles des textes traditionnels, «féminisants», tandis que les femmes «masculines» devaient s'identifier avec et juger plus favorablement les modèles des textes «non féminisants». Les femmes «féminines» devaient juger plus favorablement l'influence de la presse féminine après la lecture des articles traditionnels, tandis que les femmes «masculines» devaient juger plus favorablement l'influence de la presse féminine après la lecture des articles «modernes» contre-stéréotypés. On s'attend donc à observer une interaction entre l'effet du genre et l'effet de la version de l'article.

\section{Résultats: analyse détaillée}

54 La structure du plan comprend deux facteurs emboîtés à deux modalités:

55 - le facteur "genre » correspond à l'identité de genre évaluée par le questionnaire de Bem, la modalité " plutôt féminine » comprend les 25 sujets qui ont obtenu les scores les plus élevés sur le pôle féminin de l'échelle, la modalité " plutôt masculine » comprend les sujets qui ont obtenu les 24 sujets ayant obtenu les scores les plus élevés sur le pôle masculin de l'échelle ;

- le facteur «versions de l'article »: AGE / CORPS avec une modalité déclarée nettement « féminisante » et une modalité masculine déclarée nettement « non féminisante » par les juges. 
57 Nous avons procédé à des analyses de variances multivariées (MANOVA) sur ces données. Nous ne présenterons dans le cadre de cet article que les résultats portant sur notre hypothèse principale, sur l'existence d'un effet d'interaction entre l'effet du genre des sujets et la version de l'article, ceci pour chacune des échelles. Cet effet d'interaction devrait s'exprimer de la manière suivante:

58 - l'attitude des sujets féminins devrait être plus favorable à la version féminine de l'article qu'à la version masculine ;

59 - l'attitude des sujets masculins devrait être plus favorable à la version masculine de l'article qu'à la version féminine.

60 On s'attend donc à un effet d'interaction dite croisée: le sens de l'effet du facteur traitement (version de l'article) n'est pas le même selon les caractéristiques des sujets (ici, l'identité de genre).

61 Nous présentons dans le tableau 1, les moyennes pour les quatre groupes indépendants définis par la structure du plan pour chacune des variables dépendantes. Nous présentons dans les figures 1 le graphe des effets d'interaction. Le test de l'effet d'interaction pour les deux facteurs genre et version de l'article sont significatifs pour toutes les variables (tests F, 1, 48 ddl. <.001).

Tableau 1. Moyennes des six échelles pour chacun des groupes indépendants selon le genre des sujets et la version (féminisante vs non féminisante) de l'article

\begin{tabular}{|l|l|l|l|l|l|l|l|}
\hline $\begin{array}{l}\text { Identité } \\
\text { Genre }\end{array}$ & Article & att_age & att_corps & sim_age*** & sim_corps*** & $\begin{array}{l}\text { att_modèle } \\
\text { age*** }\end{array}$ & $\begin{array}{l}\text { att_modèle } \\
\text { corps*** }\end{array}$ \\
\hline FEMI+ & or_femi & 4.8 & 5.46 & 5.11 & 4.63 & 3.84 & 4.93 \\
\hline FEMI- & or_masc & 3.42 & 4 & 3.17 & 3.42 & 3.75 & 3.67 \\
\hline MASC- & or_femi & 3.5 & 2.5 & 2.33 & 3.5 & 2.25 & 2.83 \\
\hline MASC+ & or_masc & 5 & 5.83 & 3.92 & 5.33 & 5.08 & 5.5 \\
\hline
\end{tabular}

62 Note. ${ }^{* * *} p<<.001$.

Table 1. Means of the six scales for each independent group according to the subjects' gender and the version of the article (feminist vs non feminist)

63 Le tableau et les graphes mettent clairement en évidence qu'il s'agit pour toutes les variables d'un effet d'interaction croisée (les effets ne sont pas de même sens selon la modalité du groupe genre), 
Figure 1. Graphes d'interaction des facteurs «genre» et «version»
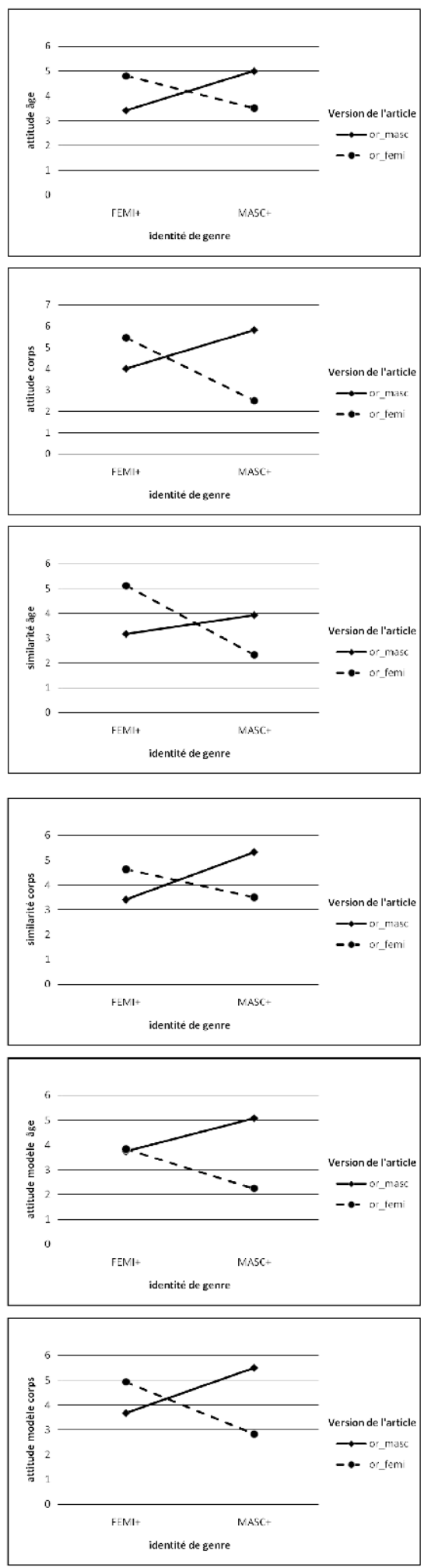

Figure 1. Interaction graphs: gender/article orientation «fem. or masc.»

excepté pour la variable «modèle âge» où l'effet est faible pour la modalité féminin de la variable genre. Pour prolonger les analyses, nous avons procédé à des comparaisons post hoc à l'aide du test de Scheffé, tous les effets de la version de l'article à l'intérieur de 
chacune des modalités de la variable genre sont significatifs à $p<.05$, excepté pour la modalité féminin de la variable modèle âge.

Ces résultats confirment bien les hypothèses. Les femmes «masculines» ont bien davantage apprécié les articles d'orientation contre-stéréotypée «non féminisante». Les réponses au questionnaire des femmes «masculines» étaient négatives envers la version traditionnelle et envers le rôle de la presse féminine en général. À l'opposé, les femmes «féminines» ont apprécié davantage les articles d'orientation féminisante et celui du rôle de cette presse.

On note toutefois qu'à une occasion les deux groupes de participantes «féminines» apprécient de façon indifférenciée et assez faiblement (notes proches de la moyenne) les deux versions opposées des articles proposés, de la même manière, contrairement aux «masculines» lorsqu'il s'agit de prendre position sur les valeurs de «modèle de la féminité» dans l'article consacré au corps.

En revanche, les femmes «masculines» maintiennent toujours leur positionnement. Elles ont une «orientation attitudinale», classique chez les minoritaires. Elles sont réactives, notamment après l'exposition aux articles contraires à leur orientation de genre même si certains textes réduisent cet écart (similarité âge).

\section{Discussion}

Faut-il interpréter le phénomène d'hésitation à prendre position à propos de la question «modèle» (âge) chez les «féminisantes»? On pourrait revenir à ce propos sur la distinction entre catégorisations sociales et stéréotypes, proposée plus haut. Les participantes d'orientation psychologique clairement «féminine», minoritaires chez ces étudiantes, peuvent parfois apprécier (tâche de listage d'idées non rapportée ici) presque aussi bien les articles avec des contre-stéréotypes non conformes à leur orientation psychologique, et ne pas se prononcer comme ici sur la valeur actuelle de «modèle» de certains textes. Elles auraient peut être commencé à intégrer l'usage régulateur de la presse féminine contemporaine depuis les années 60 , au moins à titre de modèle normatif idéal qui propose des représentations de personnages féminins dans des fictions, plus ou moins réalistes, conciliant des options comportementales, cognitives et affectives différentes ou même opposées, et ce dans un même média comme Elle (cf.chabrol, 1971).

Il faudra toutefois vérifier ultérieurement qu'elles acceptent aussi progressivement le modèle «non féminisant» à côté du «féminisant», parce qu'elles supposeraient cette conjonction plus conforme pour la majorité des jeunes femmes de leur groupe social. Elles ressentiraient que leur orientation psychologique «féminine», trop privilégiée, les place en position minoritaire face à la majorité psychologiquement «androgyne» de leur milieu étudiant qui devrait sans doute mieux adhérer à la conjonction mythique des contraires 9 , mais tout ceci reste à vérifier dans de nouvelles recherches, indispensables.

70 À l'opposé, les «masculines», également minoritaires (comparées au nombre des «androgynes» au BSRI), en refusant explicitement cette régulation conciliatrice, pour elles «imaginaire», ne voudraient pas participer à cette consommation collective de mythes régulateurs. Elles adoptent clairement une position contre-idéologique qui peut les mener à un rejet, souvent seulement verbal, d'une presse qu'elles connaissent et pratiquent de fait, parce qu'elles y trouvent aussi les éléments qui confortent leur attitude, comme on le voit bien ici même. 


\section{Conclusion}

7

Plus généralement, une orientation exclusive vers le stéréotypage traditionnel «féminisant» tendrait certes à disparaître progressivement dans le jugement social explicite et public des acteurs sociaux de cette classe sociale comme dans la presse et les médias généralistes d'information. Il est ici le fait d'une minorité réduite mais la catégorisation sociale sous-jacente traditionnelle pourrait se maintenir implicitement malgré l'évolution des comportements et des institutions. Des spécialistes du domaine soulignent que si l'on recherche une évaluation directe des catégories de genre avec des échelles de traits comme celle du BSRI en demandant la désirabilité d'une qualité psychologique pour un homme et une femme, on arrive à la conclusion qu'il y a nettement moins de catégorisation explicite, donc de stéréotypage, qu'auparavant. Cependant, des méthodes indirectes où les sujets ne perçoivent plus l'objet de la recherche (sans saillance) montrent qu'il y aurait bien moins de changements que l'on ne le pense dans les catégorisations psychologiques profondes de genre. Les orientations traditionnelles se maintiendraient malgré les importants changements sociaux, professionnels, économiques et juridiques.

72 Comment expliquer, a contrario, que le stéréotypage de la féminité traditionnelle, certes mêlé aux contre-stéréotypes les plus différents, soit encore explicitement présent - dans ses dimensions positives - dans la presse féminine et dans la publicité que lisent les femmes de toutes les classes sociales?

Il faudrait décidemment prendre acte des fonctions fort différentes du jugement public ordinaire et de celui attribué aux genres discursifs: publicitaire ou mise en scène de la mode ou des soins de beauté dans la presse féminine. On peut faire l'hypothèse d'une autonomie dans la scène publique des genres médiatiques et des sous-genres publicitaires et de médiatisation de la mode vestimentaire et des soins du corps comme des mises en scènes de la vie de femmes, souvent célèbres. Les discours de cette presse féminine doivent construire de la signification socialement partageable par toutes les femmes, soit de l'imaginaire discursif social significatif pour les objets, services, lieux, activités, audelà de leur stricte valeur d'usage fonctionnelle. Pour ce faire, ils convoquent toutes les représentations de genre supposées partageables, donc connues, traditionnelles ou non, sans véritablement les prendre en charge au plan de l'énonciation, mais en les inscrivant dans une spectacularisation discursive et iconographique, qui constitue peut-être une partie importante de leur apport, en conjoignant à des fins de régulation cognitivoaffective les représentations problématiques d'une féminité «androgyne», de fait biorientée.

74 À une époque de grands changements en Occident des rôles sociaux de la femme, et ce dans toutes les classes sociales, attribuer à cette presse une fonction «mythique» régulatrice et conciliatrice des oppositions conflictuelles au sens que Lévi-Strauss donnait à ce terme dans l'Anthropologie structurale (1958) paraît plus plausible qu'une simple fonction idéologique conservatrice qui serait confrontée à des tendances minoritaires contre-idéologiques mal contenues. Une telle hypothèse aurait au moins le mérite d'expliquer le mélange dans un même magazine d'orientations tout à fait opposées, ce qui n'est pas le cas dans la presse politique. 


\section{BIBLIOGRAPHIE}

Bem, S. L. (1974). The measurement of psychological androgyny. Journal of Consulting and Clynical Psychology, 42, 155-162.

BIBA (2006), 135, 78-82.

BIBA (2006), 114, 144-145.

Brown, M. E. (1990). Feminist cultural television criticism - culture, theory and practice. In M.E. Brown (Ed.), Television and women's culture: The politics of the popular. London: Sage.

Busby, L. J. (1975). Sex-role research on the mass media. Journal of Communication, 25, 107-131.

Butler, J. (2005). Trouble dans le genre. Pour un féminisme de la subversion. Paris: La Découverte.

Chabrol, C. (1971). Le récit féminin. Contribution à l'analyse sémiotique du courrier de cœur dans les entrevues ou «enquêtes» sur la femme dans la presse féminine actuelle. La Haye/Paris: Mouton.

Chabrol, C. (2007). Catégorisation de genre et stéréotypage: du procès des médias aux processus socio-médiatiques. In H.Boyer (éd.), Stéréotypage, stéréotypes: fonctionnements ordinaires et mises en scène, t. 1: Média(tisation)s (pp.61-72). Paris: L’Harmattan.

Chabrol, C., \& Radu, M. (2008). Psychologie de la communication et persuasion. Théories et applications. Bruxelles: De Boeck.

Chabrol, C., \& Vrignaud, P. (2006). Enquête psycho-langagière sur les effets de l'humour en publicité. Questions de communication,10, 135-156.

Chaiken, S., \& Maheswaran, D. (1994). Heuristic processing can bias systematic processing: Effects of source credibility, argument ambiguity, and task importance on attitude judgement. Journal of Personality and Social Psychology, 66(3), 460-473.

Charaudeau, P. (1994). Le contrat de communication de l'information médiatique. Le Français dans le monde ( $\mathrm{n}^{\circ}$ spécial: Médias: faits et effets), 8-19.

Debras, S. (2003). Lectrices oubliées au quotidien, Réseaux, 21(120), 177-204.

Dominick, J. R. (1979). The portrayal of women in prime time, 1953-1977. Sex Roles, 5(4), 405-411.

Eagly, A. H., \& Diekman, A. B. (1997). The accuracy of gender stereotypes: A dilemma for feminism. Revue Internationale de Psychologie Sociale, 10, 11-30.

Elle. (2006). 27, 133-134.

Epstein, L. K. (1978). Women and the news. New York: Hastings.

Ferguson, M. (1983). Forever feminine. Women's magazines and the cult of feminity. Aldershot: Gower.

Friedan, B. (1963). The feminine mystique. New York: Norton.

Ghiglione, R., \& Chabrol, C. (2000). Contrats de communication: stratégies et enjeux

(Introduction). Revue Internationale de Psychologie Sociale ( ${ }^{\circ}$ spécial), 4, 7-15.

Goffman, E. (1977). La ritualisation de la féminité. Actes de la Recherche en Sciences Sociales, 14, 34-50.

L'orientation scolaire et professionnelle, 39/3 | 2013 
Guyon, F. (1984). La publicité n'affiche pas la couleur. Paris: Denoël.

Hall, S. (1980). Cultural Studies: Two paradigms. Media, Culture and Society, 2, 57-72.

Herne, C. (1993). La définition sociale de la femme à travers la publicité. Paris: L'Harmattan.

Hobson, D. (2003). Soap Opera. Cambridge, Malden (MA): Polity.

Jaggar, A. M. (1983). Political philosophy and human nature. In A.M. Jaggar (Ed.), Feminist politics and human nature (pp.15-23). London: Rowman \& Littlefield.

Levy-Strauss, C. (1958). Anthropologie structurale. Paris: Plon.

Louis, M.-V. (1997). À propos du sexisme dans la publicité. Alternatives non violentes, 103, 29-38.

Marie France. (février 2006). 87-90.

Morin, E. (1972). Les stars. Paris: Seuil. Morley, D. (1980). The nationwide audience. London: British Film Institute.

Mouffe, C. (1992). Féminism, citizenship and radical democratic politics. In J.Butler \& J.Scott, (Eds), Feminist theorize the political (pp.369-384). New York \& London: Routledge.

Oprescu, M. (2008). Genre et médias: des mouvements féministes aux recherches sociologiques et psychologiques. Étude en réception sur l'évaluation des discours beauté/jeunesse de la presse féminine par des femmes au «genre psychologique» différent. Thèse de doctorat de $3^{\mathrm{e}}$ cycle, Paris 3.

Perret, J. B. (2003). L'approche française du genre en publicité. Réseaux, 21(120), 148-173.

Petty, R. E., \& Cacioppo, J. T. (1986). The Elaboration Likelihood Model of Persuasion. Advances in Experimental Social Psychology, 19, 123-205.

Plante, J. (2004). Le public féminin, victime des médias? Le cas des consommatrices de films pornographiques. MEI, 20, 117-127.

Press, A. (1989). Class and gender in the hegemonic process: Class differences in women's perceptions of television realism and identification with television characters. Media Culture and Society, 11(2), 229-251.

Radway, J. A. (1987). Reading the romance: Women, patriarchy, and popular literature. London: Verso.

Shields, V. R., \& Heinecken, D. (2002). Measuring up: How advertising affects self-image. Philadelphia: University of Pennsylvania Press.

Snyder, M. (1981). On the self-perpetuating nature of social stereotypes. In D.L. Hamilton (Ed.), Cognitive processes in stereotyping and intergroup behavior (pp.183-212). Hillsdale, NJ: Erlbaum.

Steeves, L. H. (1987). Feminist theory and media studies. Critical Studies in Mass Communication, 4 (2), 95-135.

Van Zoonen, L. (1991). Feminist perspectives on the media. In J.Curran \& M.Gurevitch (Eds), Mass Media and Society. London: Edward Arnold.

\section{NOTES}

1. Nous remercions Pierre Vrignaud, Professeur à Paris 10 de sa participation à la mise au point des calculs statistiques.

2. À l'époque, le travail féminin dans les classes moyennes n'était pas encore parfaitement légitimé même si de plus en plus répandu chez les plus jeunes adultes (avant 35 ans). Elle, comme d'autres magazines destinés d'abord aux classes moyennes, y accomplissait une innovation 
idéologique en posant l'équivalence de tous les «choix» de la femme. Ainsi était d'abord affirmé que chacune pouvait conjoindre dans sa vie ou même sa journée à la fois les compétences plus masculines liées au travail et celles plus féminines requises par celles de sa vie privée en couple dans son foyer, tout en suggérant que finalement même le bureau pouvait permettre de maintenir une position féminine et inversement que les travaux ménagers, cette fois choisis et non imposés, permettaient eux aussi un accomplissement finalement équivalent à celui de toute autre profession. Bref, une véritable neutralisation «mythique» de tous les conflits ou controverses liés aux changements sociaux en cours à l'époque.

3. Edgar Morin (1972) avait déjà remarquablement analysé l'importance des stars en particulier féminines pour la mythologie moderne et, en effet, les vedettes féminines symbolisent en même temps le triomphe de la plus grande féminité possible et du plus grand succès professionnel et social liés habituellement à la masculinité.

4. Il suffit souvent de réunir une série de citations supposées exemplaires.

5. Le féminisme libéral, notamment, qui a eu la plus grande influence sur les études des médias aux États-Unis (Jaggar, 1983). Le féminisme libéral s'efforce de promouvoir ou de changer des lois pour offrir aux femmes des opportunités de développement intellectuel et professionnel et pour leur assurer des conditions sociales d'égalité et d'équité. Les théoriciens féministes libéraux soutiennent l'idée selon laquelle l'inégalité serait une question des préjugés sociaux envers les femmes, qui peut être résolue à travers une argumentation rationnelle en identifiant et en montrant les éléments ou les aspects supposés responsables de ces préjugés.

6. Les analyses de contenu entreprises dans les années 70 "prouvaient» une présentation «fortement» stéréotypée des rôles de genre à travers les médias.

7. Pour quelles raisons non économiques, les publicitaires prendraient-ils ce risque de décalage à moins de reconnaître que ce sont simplement les représentations les plus partagées. Il faudrait alors que cette critique attribue ce décalage à une autre cause plus profonde que l'action de la presse, ce qui la conduit explicitement ou non vers les problématiques marxistes ou critiques radicales contemporaines.

8. BIBA, 2006, $N^{\circ} 114,144-145 ;$ BIBA, (2006), $N^{\circ} 135,78-82$; ELLE (novembre 2006), 27, 133-134; Marie France (février 2006), p.87-90.

9. Les résultats du test du BSRI montraient clairement que dans ce milieu étudiant: les positions «féminines» et « masculines» étaient ensemble minoritaires face à la majorité des «androgynes».

\section{RÉSUMÉS}

La presse féminine soulève plus de procès que d'études systématiques en sciences humaines. Elle est la cible des courants critiques marxistes ou féministes. On lui impute une influence négative, mais sans les travaux nécessaires en réception. Elle est supposée ignorer les problèmes de la femme moderne et provoquer son aliénation au bénéfice des industries de l'apparence (corps et mode) et du capitalisme. Néanmoins, ces procès n'entament pas son succès! Que faire ? Sans doute revoir les choses de plus près. Il s'agirait d'une mythologieefficace, qui ne pousse certes pas au combat militant mais qui permettrait au plus grand nombre de survivre dans ces temps de changement social du rôle de la femme. Pour s'y opposer, il faudrait peut-être en comprendre les ressorts et les bénéfices cognitifs et affectifs. 
Feminine press arouses more trials than systematic observations in social science. It is the target of critical Marxist or feminist movements. A negative influence is attributed to it, in absence of reception analysis. It is supposed to ignore true problems of modern women, and to cause their alienation to the benefit of appearance industries (body and fashion) and of capitalism. Nevertheless, these trials don't affect its success! What should be done? Certainly, make a closer analysis of it. It could be considered as an efficient mythology, which doesn't encourage militant fight, but which helps many individuals to survive in these times of social change in women's role. In order to fight against the press, one should understand the reasons and the cognitive and affective benefits of its use.

\section{INDEX}

Mots-clés : Analyses de contenu, Catégorisation, Cultural studies, Étude en réception, Féminismes, Presse féminine, Stéréotypes

Keywords : Content analysis, Feminine press, Feminisms, Reception analysis

\section{AUTEURS}

\section{CLAUDE CHABROL}

est Professeur émérite de psychologie, Paris 3. Thèmes de recherche : Analyse du discours, médias, publicité, psychologie sociale du langage. Contact : Paris 3 - UFR de communication - 1 rue de Censier - Paris cedex 05. Courriel : c.chabrol@wanadoo.fr.

\section{MIHAELA OPRESCU}

est Docteure en communication, Paris 3. Thèmes de recherche : études de genre, presse féminine, analyse en réception. 